\title{
Self-Stabilized Nonlinear Lateral Modes of Broad Area Lasers
}

\author{
DAVID MEHUYS, STUDENT MEMBER, IEEE, ROBERT J. LANG, MEMBER, IEEE, MICHAEL MITTELSTEIN, \\ JOSEPH SALZMAN, AND AMNON YARIV, FELLOW, IEEE
}

\begin{abstract}
The lateral modes of broad area lasers are investigated theoretically. The nonlinear interaction between optical field and effective refractive index leads to a saturable nonlinearity in the governing field equation, so that self-modulated solutions are found to be stable with increased current injection above saturation intensity. We derive approximate analytical solutions for traveling wave fields within the broad area laser. The field amplitude consists of a small ripple superimposed on a large de value. Matching fields at the boundary determines the modulation depth and imparts an overall phase curvature to the traveling wave mode. There are multiple lateral modes for a given set of operating conditions, and modes with successively more lobes in the ripple have greater overall phase curvature. In contrast to the linear problem, several lateral modes can achieve the same modal gain, for a given injected current density, by saturating the gain to different extent. Thus, these modes would exhibit slightly different optical powers.
\end{abstract}

\section{INTRODUCTION}

$I^{2}$ N PRINCIPLE, the most direct way to increase the optical power available from a semiconductor laser is to increase the volume of the lasing mode. It is not sufficient merely to increase the pump level because the resonator facets are susceptible to catastrophic damage at high optical powers. The simplest practical recourse is to increase the lateral dimension. In the past, stripe geometry lasers wider than about $10 \mu \mathrm{m}$ exhibited filamentary near fields that were not stable with respect to increased current injection and gave rise to equally unstable far-field patterns. Thus stripe geometry lasers were fabricated to support only a single "filament," [1] and the high-power effort shifted toward building phased arrays of such lasers. It was difficult, however, to fabricate arrays in which adjacent elements were coupled in-phase. In fact, twinlobed far-field patterns were the rule rather than the exception, because the preferred lasing mode in uniform arrays is not the fundamental supermode [2] of the array. Subsequently, many schemes have been proposed to favor the fundamental supermode, and several groups have re-

Manuscript received February 23, 1987; revised June 11, 1987. The research described in this work was conducted in the Department of Applied Physics, California Institute of Technology, and was supported in part by the Office of Naval Research and by the National Science Foundation.

D. Mehuys, M. Mittlestein, and A. Yariv are with the Department of Applied Physics, California Institute of Technology, Pasadena, CA 91125.

R. J. Lang is with the Standard Elekrik Lorenz Research Centre, 7000 Stuttgart 40, West Germany.

J. Salzman is with Bell Communications Research, Red Bank, NJ 07701. IEEE Log Number 8716666. ported laser arrays oscillating into a single-lobed far field [3]-[13]. However, all of the above require an additional degree of complexity in array design and fabrication, and true single lateral mode operation remains difficult to achieve over a large range of injected current density. More elaborate schemes such as unstable resonator geometries [14] show promise but are still technologically immature.

Recently, our group demonstrated [15] wide ( $\geq 100$ $\mu \mathrm{m})$ uniform gain broad area quantum well lasers that oscillate coherently into a nearly diffraction-limited singlelobed far-field pattern. The emission was stable over a large range of injected current, and a gradual broadening of the far field with increasing power level was the only apparent degradation. The near field was characterized by a relatively flat amplitude with a small ( $\simeq 10$ percent) superimposed ripple; the ripple period was close to $10 \mu \mathrm{m}$. Such a field cannot be explained by the simple linear theory of gain-guided structures. This led to renewed interest on our part in the basic properties and expected performance of such devices. In this paper, we investigate the theoretical behavior of these broad area lasers.

In particular, we intend to characterize the optical modes consistent with a heavily saturated gain profile. Our devices were driven up to 60 times threshold [16], so we are most interested in characterizing the high-power regime. In this regime, the intensity dependence of the carrier density and the resulting changes in the refractive index must be included in the analysis. They provide the positive feedback between the field and the material which forms its confining waveguide: It is well-known that this feedback is the basis of the regenerative self-focusing mechanism that leads to filamentation [17]. In regions of high optical intensity, the local gain is depressed by stimulated emission. Through the band-edge effect, this depression leads to a local increase in refractive index, which tends to further confine the light and increase the local field intensity. Thompson, in 1972, analyzed optical nonlinearities due to carrier depletion and found them to introduce a third-order nonlinearity in the field equation [17]. His solutions in unbounded media were either solitary "filaments," or periodic solutions consisting of adjacent "filaments" coupled either in phase or in antiphase. A serious limitation of his approach was the restriction to real refractive index variations only. As he pointed out, this restriction makes it impossible to match 
his multifilament solutions to lossy boundaries, and thus excludes the eigenfunctions of gain-guided lasers (which we introduce).

Since then, much of the work in the literature [18]-[21] has focused on analyzing the stability of solitary filaments and providing design guidelines [22] for narrow stripegeometry lasers. In this work, we focus on the "multifilament" solutions which are important in broad area lasers. However, since the modulation depth of our solutions is relatively small, it is inaccurate to characterize these solutions as "coupled filaments." In fact, to the contrary, these modes evolve in a smooth way from higher order modes of the complex linear waveguide. Further, we show that gain saturation is responsible for the stabilization of "filament" size. Lateral carrier diffusion does not play a significant role in this stabilization.

\section{Carrier-Dependent Refractive Index}

In this section the complex refractive index is written to include the carrier dependence. Throughout this work we consider traveling waves of the form

$$
\mathcal{E}(x, y, z, t)=E(x) F(x, y) e^{i(\beta z-\omega t)}
$$

where $x$ is the lateral dimension, $y$ the transverse dimension, and $z$ the direction of propagation. The propagation constant is $\beta$ and the angular frequency $\omega . E(x)$ is the unknown lateral mode, and $F(x, y)$ gives the transverse mode field distribution. As the problem cannot be solved exactly by separation of variables, the transverse mode shape contains a slowly-varying dependence on the lateral coordinate [23].

Under these approximations, the lateral mode $E(x)$ satisfies the Helmholtz equation

$$
\frac{d^{2} E(x)}{d x^{2}}+k_{0}^{2}\left\{n_{\text {eff }}^{2}(x)-\eta^{2}\right\} E(x)=0,
$$

where $n_{\mathrm{eff}}^{2}(x)$ is the usual effective index determined by solving the transverse eigenmode problem at each value of $x$. The eigenvalue, $\eta^{2}$, is defined by the longitudinal propagation constant $\beta=k_{0} \eta$, and $k_{0}$ is the free-space propagation constant.

The physical origin of the self-focusing mechanism lies in the local depression of the gain profile by stimulated emission (spatial hole-burning), and this is what we shall quantify first. It will then be incorporated into the effective refractive index. Fig. 1(a) shows a broad area device of the type we shall consider. The laser shown is a single quantum well separate confinement heterostructure known to give very low threshold densities [24]. Fig. 1(b) and (c) show representative optical modes of the transverse and lateral waveguides, respectively. The transverse mode is index-guided, and for the single quantum well of thickness $L_{y}$, it is characterized by a small optical confinement factor $\Gamma$.

The lateral waveguide is formed by the current stripe and the lateral mode depends on the gain and index profiles introduced by the steady-state carrier distribution. In

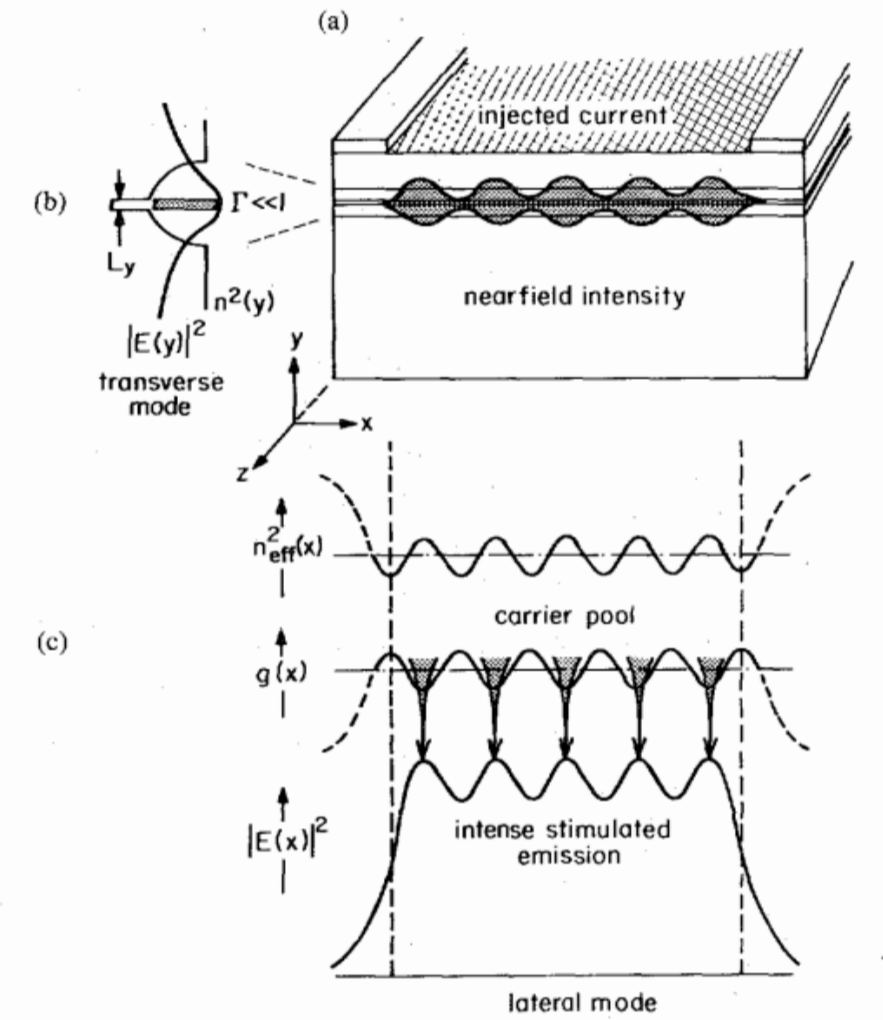

Fig. 1. (a) Stripe-geometry broad area laser with coordinate system used in this analysis. (b) Fundamental transverse mode of graded index single quantum well structure, showing very small optical confinement factor. (c) Nonlinear lateral mode as observed from our best devices. The gain is locally depleted by stimulated emission in the high intensity regions, leading to an increase in the local refractive index and the phenomenon of self-focusing.

the carrier rate equation we shall consider one-dimensional variables, since we have separated out the longitudinal dependence and integrated out the transverse dependence. When the lateral mode "sees" an effective index $n_{\text {eff }}$, the steady-state rate equation becomes [19], [20]

$$
\begin{aligned}
\frac{J(x)}{e}= & \left(\frac{c}{n_{\mathrm{eff}}}\right) \Gamma g(N(x)) P(x) \\
& +\frac{N(x)}{\tau_{\mathrm{sp}}}-D \frac{d^{2} N(x)}{d x^{2}}
\end{aligned}
$$

where

$$
\begin{aligned}
J(x)= & \text { injected current density }\left[\mathrm{cm}^{-2} \mathrm{~s}^{-1}\right] \\
N(x)= & \text { carrier density }\left[\mathrm{cm}^{-2}\right] \\
P(x)= & \text { photon density in lateral mode } E(x)\left[\mathrm{cm}^{-2}\right] \\
g(x)= & \text { spatial gain profile in the quantum well } \\
& {\left[\mathrm{cm}^{-1}\right] } \\
\tau_{\mathrm{sp}}= & \text { spontaneous lifetime }[\mathrm{s}] \\
D= & \text { lateral diffusion coefficient }\left[\mathrm{cm}^{2} \mathrm{~V}^{-1} \mathrm{~s}^{-1}\right] .
\end{aligned}
$$

Equation (3) states that at a given position $x$, the carriers gained by injected current are balanced in steady state by losses due to stimulated and spontaneous emission and by 
lateral diffusion. Nonradiative recombination processes can be lumped into the spontaneous emission lifetime $\tau_{\mathrm{sp}}$. We define a normalized saturated gain profile as

$$
\gamma(x) \equiv \frac{g(x)-g_{\text {th }}}{g_{\text {th }}}=\frac{g_{\text {th }}^{\prime}}{g_{\text {th }}}\left(N(x)-N_{\text {th }}\right) .
$$

where, as is customary [17], we have linearized about the threshold gain $g_{\mathrm{th}} \equiv g\left(N_{\text {th }}\right) . g_{\text {th }}^{\prime}$ is the differential gain (with respect to carrier density) at threshold. The dimensionless quantity $\gamma(x)$ quantifies the spatial hole-burning, giving the deviation from threshold gain in units of the threshold gain. Equation (3) can now be expressed as a second-order linear ordinary differential equation in the normalized saturated gain profile:

$$
\begin{gathered}
L_{\mathrm{sp}}^{2} \frac{d^{2} \gamma(x)}{d x^{2}}-\left[1+\frac{P(x)}{P_{\text {sat }}}\right] \gamma(x) \\
=\frac{P(x)}{P_{\text {sat }}}-\frac{J(x)-J_{\text {th }}}{J_{\text {sat }}},
\end{gathered}
$$

where the following definitions have been made:

$$
\begin{gathered}
L_{\mathrm{sp}}^{2} \equiv D \tau_{\mathrm{sp}} \\
P_{\mathrm{sat}} \equiv \frac{n_{\mathrm{eff}} / c}{\Gamma g_{\mathrm{th}}^{\prime} \tau_{\mathrm{sp}}} \\
J_{\mathrm{th}} \equiv \frac{e N_{\mathrm{th}}}{\tau_{\mathrm{sp}}} \\
J_{\mathrm{sat}} \equiv \frac{e g_{\mathrm{th}}}{\tau_{\mathrm{sp}} g_{\mathrm{th}}^{\prime}} .
\end{gathered}
$$

$L_{\mathrm{sp}}$ is the diffusion length when the carrier lifetime is determined solely by spontaneous emission and $J_{\text {th }}$ is the threshold current density. The equation has no simple solution, as the diffusion operator $\boldsymbol{L}$ is $\boldsymbol{x}$-dependent:

$$
\boldsymbol{L}(x) \equiv L_{\mathrm{sp}}^{2} \frac{d^{2}}{d x^{2}}-\left[1+\frac{P(x)}{P_{\mathrm{sat}}}\right],
$$

and $P(x)$ is still unknown. We can, however, find an approximate solution in terms of $P(x)$. Since $P(x) \geq 0$ for all $x$, it is appropriate to find a WKB approximation for the Green's function of $\boldsymbol{L}(x)$ [25]:

$$
\begin{aligned}
& G_{W K B}\left(x, x^{\prime}\right) \\
& =\frac{1}{2 L_{\mathrm{sp}}} \frac{\exp \left\{-\frac{1}{L_{\mathrm{sp}}}\left|\int_{x^{\prime}}^{x} \sqrt{1+\frac{P(t)}{P_{\text {sat }}}} d t\right|\right\}}{\left[\left(1+\frac{P(x)}{P_{\text {sat }}}\right)\left(1+\frac{P\left(x^{\prime}\right)}{P_{\text {sat }}}\right)\right]^{1 / 4}} .
\end{aligned}
$$

The Green's function is integrated against the right-hand side of (5) and the integral is expanded asymptotically in powers of the diffusion length. The result for the saturated gain profile is as follows:

$$
\begin{gathered}
\gamma(x) \sim \gamma_{0}(x)+\frac{L_{\mathrm{sp}}^{2}}{1+\frac{P(x)}{P_{\mathrm{sat}}}} \frac{d^{2} \gamma_{0}(x)}{d x^{2}} \\
+O\left\{\frac{L_{\mathrm{sp}}^{4}}{\left(1+\frac{P(x)}{P_{\mathrm{sat}}}\right)^{2}}\right\}
\end{gathered}
$$

where

$$
\gamma_{0}(x)=\frac{\frac{J(x)-J_{\text {th }}}{J_{\text {sat }}}-\frac{P(x)}{P_{\text {sat }}}}{1+\frac{P(x)}{P_{\text {sat }}}}
$$

is the solution when diffusion is neglected. Consider a saturated gain profile that reflects a self-modulated near field. While the level of the losses determines the average gain level, regions of relatively high (low) optical intensity cause regions of local depression (elevation) in the gain. This effect, due to stimulated emission, is dominant and is represented by the first term in (9). In addition, diffusion will cause some of the carriers to shift from regions of high gain to regions of low gain-filling in the gaps-and this effect is represented by the second term in (9); it is a correction to the first term.

Now, suppose that $\gamma(x)$ oscillates about zero with a periodicity defined by transverse wave vector $k_{t}$. Compared to the first term in (9), the diffusion term is of order $k_{t}^{2} L_{\mathrm{sp}}^{2} /\left(1+\left(P(x) / P_{\mathrm{sat}}\right)\right)$. If $k_{t} L_{\mathrm{sp}}$ is small compared to one, then $k_{t}^{2} L_{\mathrm{sp}}^{2} \ll 1$, and the diffusion correction is small; as the power increases over $P_{\text {sat }}$, it becomes smaller yet. That is, the diffusion length, being proportional to the square root of the carrier lifetime, is reduced in the presence of stimulated emission. Note that $P_{\text {sat }}$ can be interpreted as the photon density at which the stimulated emission rate equals the spontaneous emission rate, since at this intensity the carrier lifetime is reduced by a factor of two. This is in accordance with other definitions of the saturation intensity [17], [26].

Since modulation in $P(x)$ occurs only on spatial scales longer than $L_{\mathrm{sp}}$ and the effects of diffusion become vanishingly small at high power, we neglect them. As a result, we take (10) as the saturated gain profile. It remains to incorporate this expression into the complex refractive index. Observing the traveling wave convention (1) leads to the relationship

$$
n_{\mathrm{eff}}^{2}(x)=n_{0}^{2}-\frac{n_{0}}{k_{0}} \Gamma g_{\mathrm{th}}(b+i) \gamma(x)
$$

where $n_{0}$ is the effective index of refraction corresponding to the threshold gain level, and $b$ is the antiguiding factor [positive, with convention (1)]. In our model, $b$ is identical to the linewidth enhancement factor $\alpha$ at threshold 
carrier density. Finally, we establish the dependence of $n_{\text {eff }}^{2}$ on field $E(x)$ by making use of the following relation:

$$
|E(x)|^{2}=\frac{2 \hbar \omega}{n_{0}^{2}} \frac{\Gamma P(x)}{L_{y}}
$$

where $\hbar \omega$ is the lasing transition energy and $L_{y}$ is the previously mentioned active layer thickness. The field strength at saturation is then [from (6b) and (12)]

$$
E_{\mathrm{sat}}^{2}=\frac{2 \hbar \omega}{n_{0}^{2}} \frac{n_{\mathrm{eff}}}{c} \frac{1}{g_{\mathrm{th}}^{\prime} L_{y} \tau_{\mathrm{sp}}} .
$$

We further define

$$
\epsilon_{\mathrm{sat}} \equiv \frac{n_{0}}{k_{0}} b \Gamma g_{\mathrm{th}}
$$

and note for future reference that $\epsilon_{\text {sat }}$ is proportional to the threshold gain. Equation (11) for the carrier-dependent dielectric constant becomes

$$
\begin{aligned}
n_{\mathrm{eff}}^{2}(x)= & n_{0}^{2}+\epsilon_{\mathrm{sat}}\left(1+\frac{i}{b}\right) \\
& \cdot \frac{|E(x)|^{2}-E_{\mathrm{sat}}^{2} \frac{J(x)-J_{\mathrm{th}}}{J_{\mathrm{sat}}}}{|E(x)|^{2}+E_{\mathrm{sat}}^{2}} .
\end{aligned}
$$

Equation (15) predicts, as expected, an increase in both the real and imaginary parts of $n_{\mathrm{eff}}^{2}$ in regions where $|E(x)|^{2}$ is large. That is, the gain is decreased while the refractive index is increased. Furthermore, at threshold, $J=J_{\text {th }}$ and $P=0$, which gives $n_{\mathrm{eff}}^{2}=n_{0}^{2}$ as desired. At low-field intensity, $|E(x)|^{2} \ll E_{\text {sat }}^{2}$, this expression is of the form common to the nonlinear optics literature [27]: $n^{2}(x)=n_{0}^{2}+n_{2} \cdot I(x)$, where $I$ is the optical intensity. However, in our case, the nonlinearity in the effective index is saturable. As seen from (15) the maximum local increase in dielectric constant occurs in the saturated limit $|E(x)|^{2} \gg E_{\text {sat }}^{2}$, and is given by $\epsilon_{\text {sat }}$. It can be appreciated from (14) that the parameter $\epsilon_{\text {sat }}$ represents the depression in dielectric constant in pumping from transparency to threshold current density. To minimize selffocusing, it should be as small as possible. Equation (14) indicates that to accomplish this, small values for the antiguiding factor, optical confinement factor, and threshold gain are desired. All of these quantities are generally regarded as smaller in quantum well (QW) lasers than in regular double heterostructure (DH) lasers, and thus we conclude that the nonlinear action is weaker in QW lasers. $\epsilon_{\text {sat }}$ has been measured in both QW and DH lasers and is known to be smaller by a factor of 2 in the case of the QW lasers [28]. In very low threshold QW lasers, this improvement may be doubled again.

\section{Complex-Valued Nonlinear Field Equation}

Incorporating the complex effective refractive index (15) into the Helmholtz equation (2) gives the following second-order, nonlinear, nonanalytic, complex eigen- value problem to be solved for the modes of the broad area laser:

$$
\begin{aligned}
\frac{1}{k_{0}^{2}} \frac{d^{2} E(x)}{d x^{2}}+\left\{n_{0}^{2}-\eta^{2}+\left(1+\frac{i}{b}\right) \epsilon_{\text {sat }}\right. \\
\left.\cdot \frac{|E(x)|^{2}-E_{\text {sat }}^{2} \frac{J(x)-J_{\text {th }}}{J_{\text {sat }}}}{|E(x)|^{2}+E_{\text {sat }}^{2}}\right\} E(x)=0
\end{aligned}
$$

As can be deduced from phase-plane arguments, (16) supports periodic solutions of the form $E(x)=E_{0}(1+$ $m e(x))$ where $E_{0}$ represents the average field amplitude and $e(x)$ is a periodic function that contains the filamentary self-modulation. Here $m$ is a modulation depth small compared to unity, so the composite solution has no nulls. Thompson identified such "multifilament" solutions in his treatise, and it is evident that corresponding solutions exist in the complex case for a laser of infinite width. For our laser of finite width, we take a solution of the form

$$
E \equiv E_{0} e^{a+i \phi}
$$

so that amplitude variations appear in $a$ and phase variations in $\phi$. As such, small modulation solutions are characterized by small $a$, in which case appropriate linearizations can be easily made. Let $\xi$ be a dimensionless position co-ordinate: $x \equiv \xi d$, where $d$ is the half-width of the laser. Since $E(0) \neq 0$, we consider only even solutions for $E(\xi)$, and thus our task is to solve the problem on the interval $0<\xi<1$. In the normalized coordinates, equation (16) becomes

$$
a^{\prime \prime}+i \theta^{\prime}+a^{\prime 2}+i 2 a^{\prime} \theta-\theta^{2}+L(a)=0
$$

where $\theta(\xi) \equiv \phi^{\prime}(\xi)$ (note that the equation depends only on the phase gradient $\theta$, not on the absolute phase $\phi$ ). The intensity-dependent refractive index term is included in the last term of (18):

$$
\begin{gathered}
L(a) \equiv k_{0}^{2} d^{2}\left\{n_{0}^{2}-\eta^{2}+\left(1+\frac{i}{b}\right) \epsilon_{\mathrm{sat}}\right. \\
\left.\cdot \frac{E_{0}^{2} e^{2 a}-E_{\text {sat }}^{2} \frac{J-J_{\text {th }}}{J_{\text {sat }}}}{E_{0}^{2} e^{2 a}+E_{\text {sat }}^{2}}\right\} .
\end{gathered}
$$

The term $L(a)$ can be linearized for the case of $|a|<<$ 1 (small modulation depth):

$$
L(a)=L_{0}+L_{1} \cdot a+O\left(a^{2}\right)
$$

with

$$
\begin{aligned}
L_{0} \equiv & k_{0}^{2} d^{2}\left(n_{0}^{2}-\eta^{2}\right) \\
& +\frac{1}{2}\left(1+\frac{i}{b}\right) k_{0}^{2} d^{2} \epsilon_{\text {sat }}(1-\sigma) \\
L_{1}= & \left(1+\frac{i}{b}\right) q_{0}^{2}
\end{aligned}
$$


and where the following definitions have been made:

$$
\begin{array}{r}
\sigma \equiv \frac{\frac{J-J_{\text {th }}}{J_{\text {sat }}}+1}{\frac{E_{0}^{2}}{E_{\text {sat }}^{2}}+1} \\
\mu \equiv \frac{E_{0}^{2}}{E_{0}^{2}+E_{\text {sat }}^{2}} \\
q_{0}^{2} \equiv 2 k_{0}^{2} d^{2} \sigma \mu \epsilon_{\text {sat }}
\end{array}
$$

Here $\sigma$ is a dimensionless quantity related to the ratio between pump and field intensities, and in our model, is constrained by energy conservation to be an $O(1)$ term. On the other hand, $\mu$ gives the approximate ratio of stimulated to stimulated + spontaneous emission, and saturates smoothly towards unity in the high-power limit. $q_{0}^{2}$ is proportional to the $\sigma \mu$ product, and (as we shall see) corresponds to the squared filament wave vector.

We have solved (18) [subject to the linearization (20)] analytically. The bulk of the derivation appears as Appendix A. At this point, we merely summarize the result.

In addition to the filamentary self-modulation, we allow for a global phase curvature and a slowly-varying amplitude variation to satisfy the loss requirement of the amplified mode. We separate the two as

$$
\begin{aligned}
& a=a_{f}+a_{s} \\
& \theta=\theta_{f}+\theta_{s}
\end{aligned}
$$

where $f$ denotes "fast" (or "filament") and $s$ denotes "slow.,

The fast and slow variables are decoupled by an appropriate averaging procedure. The fast, or self-modulation terms, are

$$
\begin{aligned}
a_{f}= & m(\xi) \cos \int q_{r} d \xi \\
\theta_{f}= & m(\xi)\left[-\frac{q_{0}^{2}}{b q_{r}}\right. \\
& \left.\cdot \sin \int q_{r} d \xi-2 \theta_{s} \cos \int q_{r} d \xi\right] \\
\phi_{f}(\xi) \simeq & m(\xi)\left[-\frac{q_{0}^{2}}{b q_{r}^{2}}\right] \\
& \left.\cdot \cos \int q_{r} d \xi-2 \frac{\theta_{s}}{q_{r}} \sin \int q_{r} d \xi\right]
\end{aligned}
$$

where $m$ and $q_{r}$ are the slowly-varying modulation depth and filament wavenumber, given by

$$
\begin{gathered}
m(\xi)=m_{0}\left(1+\rho \sinh ^{2}\left(\chi_{0} \xi\right)\right)^{1 / 2 \rho}, \\
\rho \equiv 1+\frac{4 b^{2} \chi_{0}^{2}}{q_{0}^{2}}
\end{gathered}
$$

$$
\begin{aligned}
\int q_{r} d \xi= & q_{0} \xi+\frac{2 b^{2} \chi_{0}}{q_{0}} \\
& \cdot\left(\chi_{0} \xi-\tanh \left(\chi_{0} \xi\right)\right)
\end{aligned}
$$

and $m_{0}$ is the modulation depth at the center of the device.

The slow amplitude and phase variations are

$$
\begin{aligned}
a_{s}= & \frac{b^{2} \chi_{0}^{2}}{q_{0}^{2}} \tanh ^{2}\left(\chi_{0} \xi\right) \\
& -\frac{1}{2}\left(m^{2}(\xi)-m_{0}^{2}\right) \\
\theta_{s}= & b \chi_{0} \tanh \left(\chi_{0} \xi\right) \\
\phi_{s}= & b \ln \cosh \left(\chi_{0} \xi\right) .
\end{aligned}
$$

These solutions are parameterized by $m_{0}$ and $\chi_{0}$. Later it will be shown how these quantities are related to the real and imaginary parts of the eigenvalue, $\eta$.

The parameter $\chi_{0}$ appears throughout, and we pause to discuss its significance. Locally, the angle between the optical axis and the direction of phase and energy propagation is, for small angles (in radians),

$$
\theta(\xi)=\frac{1}{\operatorname{Re}(\beta)} \frac{d \phi}{d x}=\frac{\lambda_{0}}{2 n \pi d} \theta(\xi)
$$

where $n$ is the real refractive index and $\theta=\theta_{s}+\theta_{f}$, as before. Thus $\lambda_{0} b \chi_{0} / 2 n \pi d$ is the maximum slowly-varying angle of off-axis propagation, while $d / \chi_{0}$ is the lateral position of the "knee" of the hyperbolic tangent. The value of $\chi_{0}$ is set by matching to the fields outside of the gain stripe. As a rule, the larger the change in $n_{\text {eff }}$ is, the larger $\chi_{0}$ must be to accommodate it. Note that solutions in media of infinite extent are obtained by putting $\chi_{0}=$ 0 , while $m_{0}$ is unspecified.

Thus, depending on the magnitude of $\chi_{0}$ compared to unity, the phase front can be approximately parabolic over the width of the device $\left(\chi_{0}<1\right)$, or else quickly approach a linear asymptote on either side $\left(\chi_{0}>1\right)$. The important consequence of this fact is that in the former case, the far field will be essentially single-lobed, while in the latter case a sharply-defined double-lobed far field will result. Consequently, it is desirable to minimize the change in $n_{\text {eff }}$ at the edge of the gain stripe to reduce $\chi_{0}$ and get a narrow far field.

The primary structure in the near-field pattern is the selfmodulation. Its wavenumber in the center of the laser is $q_{0}$, defined by $(22 \mathrm{c})$ as

$$
q_{0}^{2}=2 \sigma k_{0}^{2} d^{2} \epsilon_{\mathrm{sat}} \frac{E_{0}^{2}}{E_{0}^{2}+E_{\mathrm{sat}}^{2}} .
$$

This is an intensity-dependent quantity, small at low intensity, but quickly increasing to a limiting value. Hence the filament spacing becomes stable as saturation intensity is surpassed.

Since the filament width saturates, the number of filaments must also stabilize. From (24a) we see that the 
number of lobes in the near field is

$$
N=\left\lceil 1+\int_{0}^{1} \frac{q_{r}}{\pi} d \xi\right\rceil \geq\left\lceil 1+\frac{q_{0}}{\pi}\right\rceil
$$

where $\lceil\cdots\rceil$ denotes the greatest integer less than or equal to the argument. The variable $q_{r}$ is always greater than or equal to $q_{0}(25 \mathrm{~b})$, which establishes a lower bound for $N$. The squared wavenumber $q_{0}^{2}$ saturates to $2 \sigma k_{0}^{2} d^{2} \epsilon_{\text {sat }}$. Recall that $\sigma$ is an $O(1)$ quantity and can be taken as unity for purposes of discussion. As we mentioned earlier, $\epsilon_{\mathrm{sat}}$ is the difference in real dielectric constant between transparency and threshold. This difference comes from unsaturable losses (chiefly mirror losses). For a laser of width $W$, we have

$$
N \geq\left\lceil 1+W / W_{f}\right\rceil
$$

where $W_{f}$ is a saturated filament spacing given by

$$
W_{f} \equiv \sqrt{\frac{\pi \lambda_{0} L}{\operatorname{Re}\left(n_{0}\right) b[\alpha L+\ln (1 / R)]}} .
$$

where $L$ is the laser length, $R$ is the facet reflectivity, and $\alpha$ is the distributed loss constant. Thus, unsaturable losses in addition to the mirror losses decrease the saturated filament spacing and increase the number of filaments.

In order to quantify this analysis, we choose $\epsilon_{\text {sat }}$ to characterize the wafers grown in our laboratory by molecular beam epitaxy. For a single quantum well device with threshold current density $250 \mathrm{~A} / \mathrm{cm}^{2}$, the relevant values are $\Gamma g_{\text {th }}=30 \mathrm{~cm}^{-1}$ and $b=2$ [29]. The value of $b$ has been estimated for AlGaAs lasers to lie in the range 2-6 [30] with the lower values more appropriate for quantum well lasers. At $\lambda=0.845 \mu \mathrm{m}$, this gives $\epsilon_{\text {sat }}=2.7 \times$ $10^{-3}$, and a saturated filament spacing of $\sim 12 \mu \mathrm{m}$. This value justifies our decision to neglect diffusion effects in formulating (15). For a device $100 \mu \mathrm{m}$ wide, the estimated lower bound on filament number is $N=9$.

At this point, the solutions are parameterized by the modulation depth $m_{0}$, phase gradient $\chi_{0}$, and the field amplitude $E_{0}$. It remains to relate $m_{0}$ and $\chi_{0}$ to the eigenvalue, $\eta$. We make implicit definitions of $\Delta_{r, i}$ in terms of $\eta$ as follows:

$$
\begin{aligned}
k_{0}^{2} d^{2}\left(n_{0}^{2}-\eta^{2}\right) & \simeq 2 n_{0} k_{0}^{2} d^{2}\left(n_{0}-\eta\right) \\
& \equiv \frac{1}{2}\left(\Delta_{r}+i \frac{\Delta_{i}}{b}\right) .
\end{aligned}
$$

Thus, $\Delta_{r, i}$ gives the deviation of the eigenvalue from the effective index at threshold. From (21a) we have

$$
\begin{aligned}
& L_{0} \equiv L_{0}^{r}+i L_{0}^{i} \\
& L_{0}^{r}=\frac{1}{2}\left(\Delta_{r}+k_{0}^{2} d^{2} \epsilon_{\mathrm{sat}}(1-\sigma)\right) \\
& L_{0}^{i}=\frac{1}{2 b}\left(\Delta_{i}+k_{0}^{2} d^{2} \epsilon_{\mathrm{sat}}(1-\sigma)\right) .
\end{aligned}
$$

Using (A7), (A24)-(A25) (from Appendix A) leads to

$$
\begin{aligned}
& \Delta_{r}=k_{0}^{2} d^{2} \epsilon_{\mathrm{sat}}(\sigma-1)-m_{0}^{2} q_{0}^{2} \\
& \Delta_{i}=k_{0}^{2} d^{2} \epsilon_{\mathrm{sat}}(\sigma-1)-2 m_{0}^{2} q_{0}^{2}-2 b^{2} \chi_{0}^{2} .
\end{aligned}
$$

Thus, solving for the physically meaningful quantities of modulation depth $m_{0}$ and the slow phase gradient $\chi_{0}$ by boundary matching is equivalent to determining the eigenvalues $\Delta_{r}$ and $\Delta_{i}$.

A physically important parameter is the modal gain $G_{m}$, defined to be the rate at which the traveling wave solution grows. For purposes of discussion, we allow the modal gain to be different from the threshold gain $\Gamma g_{\text {th }}$ and thus examine all traveling wave solutions. Since $G_{m}-\Gamma g_{\text {th }}=$ $-2 k_{0} J m\left(\eta-n_{0}\right)$, we have

$$
G_{m}=\Gamma g_{\text {th }}+\frac{\Delta_{i}}{2 b \operatorname{Re}\left(n_{0}\right) k_{0} d^{2}} .
$$

Ultimately, energy conservation requires $\Delta_{i}=0$ for a lasing mode, and this determines the unknown field amplitude $E_{0}$ in terms of $m_{0}$ and $\chi_{0}$ via (34b). The three terms in $\Delta_{i}$ correspond to three physical mechanisms that affect the gain. The first is proportional to $\sigma-1$. Recall that $\sigma$ is related to the ratio of pump intensity to field intensity. An increase in $\sigma$ indicates an increase in the pump level (or decrease in the optical power) which reduces the amount of gain saturation. The second term, proportional to $m_{0}^{2}$, reflects the inefficiency introduced by modulation in the near-field pattern. In comparison of a field with a modulated near field to one without, where both experience the same modal gain, the average gain level consistent with the modulated field is increased by an amount proportional to the square of the modulation depth; that amount appears here. The third term, proportional to $\chi_{0}^{2}$, reflects the losses due to phase curvature, or off-axis propagation. Energy propagating at an angle to the optical axis is absorbed by the lossy boundaries; this effect also reduces the gain.

\section{The Outer Solution and Boundary Matching}

Beyond the gain stripe ( $\xi \geq 1$ ), the pump current is zero. However, several processes conspire to prevent the gain from immediately taking on its unpumped value. Current spreading will cause the current injected into the active region to taper off at the edge of the gain stripe. Optical pumping also occurs, which partially bleaches the unpumped material. Our model of $n_{\text {eff }}$ is based on a linearization of the gain about the threshold gain, and extension of this model into the unpumped region would be clearly inaccurate. In addition, the roll-off rate of $n_{\text {eff }}$ would be of the same order of magnitude as $q_{0}$. Consequently, the separation of scales we used in the previous portion of this paper would be inappropriate.

However, the exact shape of the gain distribution outside the gain stripe affects only slightly the rate at which the field grows or decays at the edge of the gain stripe, which determines the values that the inner solution must take on there. For our model, we will assume that the 
effective index of refraction outside the stripe has a functional form that satisfies the following criteria:

1) $n_{\text {eff }}=n_{0}$ at the edge of the gain stripe.

2) $n_{\text {eff }}$ rolls off smoothly to its full absorption value, defined to be $n_{1}$.

In addition, we would like the specific functional form to allow for a closed-form analytic solution. These criteria are satisfied if we take

$$
\begin{aligned}
n_{\text {eff }}^{2} \equiv & n_{0}^{2}+B \tanh \kappa(\xi-1) \\
& +C \tanh ^{2} \kappa(\xi-1)
\end{aligned}
$$

where

$$
B+C=n_{1}^{2}-n_{0}^{2}
$$

and $\kappa$ characterizes the roll-off rate.

Our approximate solution outside the gain stripe is the solution of the Helmholtz equation (2) with this effective index; that is,

$$
E=E_{1} e^{-D(\xi-1)} \operatorname{sech}^{F / \kappa} \kappa(\xi-1)
$$

where the complex constants $D$ and $F$ satisfy

$$
\begin{aligned}
F^{2}+\kappa F+2 D F & =-\left(n_{1}^{2}-n_{0}^{2}\right) \\
D^{2}-\kappa F & =-\frac{1}{2}\left(\Delta_{r}+i \frac{\Delta_{i}}{b}\right) .
\end{aligned}
$$

The appropriate branches to select are $\operatorname{Re}(D) \geq 0$ and $\operatorname{Re}(F) \geq 0$. The eigenvalues are determined by the requirement that the field $E$ and its first derivative $E^{\prime}$ be continuous at the edge of the gain stripe $(\xi=1)$. Both conditions can be met by the requirement

$$
\left.\frac{E^{\prime}}{E}\right|_{\xi=1^{-}}=\left.\frac{E^{\prime}}{E}\right|_{\xi=1^{+}} .
$$

Consequently, the transcendental equation which determines the eigenvalues and modal gain is:

$$
\begin{gathered}
a^{\prime}(1)+i \theta(1)=-D . \\
\text { V. Discussion }
\end{gathered}
$$

We can now solve the eigenvalue equation for $m_{0}$ and $\chi_{0}$ and substitute the results into our expressions for the lateral mode. In Fig. 2(a) and (b) we have plotted the lateral power distribution and local phase angle, respectively, for a 10 -filament mode of a $100 \mu \mathrm{m}$ wide device, for current pumping $J=6 J_{\text {th }}, J_{\text {th }} / J_{\text {sat }}=3, b=2$, and $\epsilon_{\mathrm{sat}}=2.7 \times 10^{-3}$. The unsaturated (amplitude) loss outside the gain stripe is taken to be $90 \mathrm{~cm}^{-1}$, and $\kappa$ corresponds to a $10-90$ percent roll-off distance of $20 \mu \mathrm{m}$ in $n_{\text {eff }}$. The number of lobes in the near-field pattern, 10 , is higher than our estimate, reflecting the additional contributions to $q_{r}$ that come from $\chi_{0}$. As we said, $\chi_{0}$ is set by boundary effects; consequently, lossier boundaries on the gain stripe will increase the number of lobes in the near field. This pattern is very similar to the experimental trace reported in [15] [reproduced in Figure 2(c)] in the size

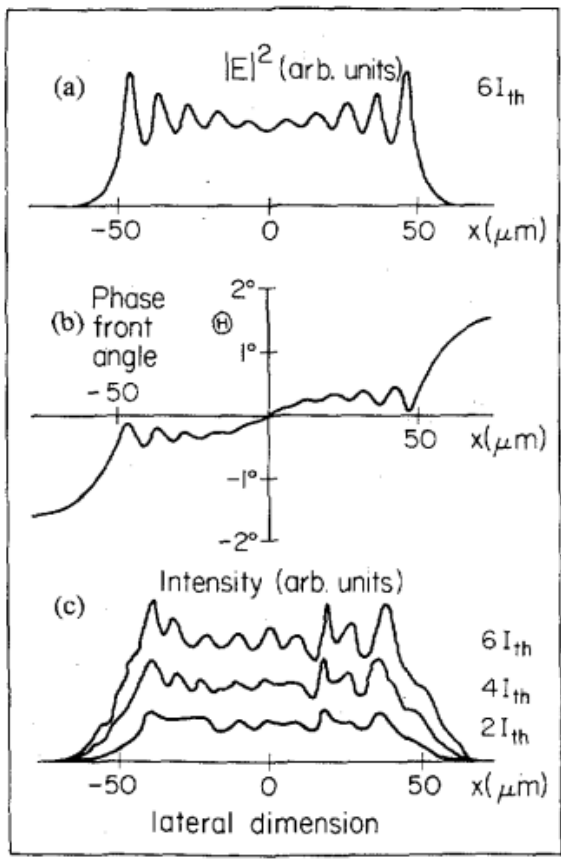

Fig. 2. Our analytical solution for the lowest- $\sigma$ 10-filament mode of a device with half-width $d=50 \mu \mathrm{m}, \epsilon_{\mathrm{sat}}=2.7 \times 10^{-3}$ and $\Gamma g_{\mathrm{th}}=30 \mathrm{~cm}^{-1}$. The spreading parameter at the edges is $\kappa^{-1}=20 \mu \mathrm{m}$. (a) Near-field intensity. (b) Local phase front angle. The slow component of the phase front angle reaches $0.32^{\circ}$ at the lossy boundary, corresponding to emission off-axis of about $1.1^{\circ}$ outside the facet. (c) Experimental trace of near-field intensity of $100 \mu \mathrm{m}$ wide device at several points above threshold [15].

and spacing of the lobes and the increase in modulation depth towards the edge of the stripe. In addition, we can estimate the width of the far field from the largest local average phase angle under the gain stripe. In Fig. 2(a), the average phase angle (inside the facet) is approximately, from (38), $0.32^{\circ}$, which yields a far-field width of $2.1^{\circ}$ outside the laser; that, too, is in agreement with [15].

Next, let us consider the lateral mode spectrum. In the linear case, this spectrum is characterized by a finite set of modes, each with a distinct modal gain. The mode with the highest modal gain is deemed to be the lasing mode at threshold. However, the situation is more complex in the associated nonlinear problem. In addition to modal gain $G_{m}$, each mode must also be characterized by a field amplitude $E_{0}$, at a given injected current density. This added degree of freedom allows a finite set of lateral modes to have the same modal gain by allowing the individual amplitudes to vary.

This multiplicity is illustrated in Fig. 3. Here we plot the modal gain as a function of $\sigma$ for modes containing 813 filaments. If the resonator losses are $30 \mathrm{~cm}^{-1}$, then all solutions with matching modal gain are candidates for lasing modes. For comparison, the gain of a uniform plane wave of the same $E_{0}$ is also plotted. None of the lateral modes is as efficient as a plane wave in extracting gain. That is to be expected because the lateral modes have the absorbing boundaries to contend with. The boundaries introduce losses in two ways. First, they induce a spatial modulation, which lowers the extraction efficiency by an 


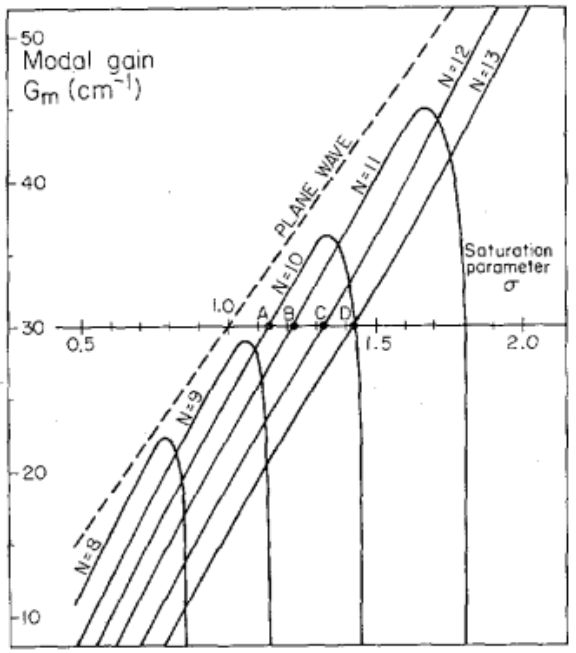

Fig. 3. Traces of the $N=8$ through 13 filament solutions in the modal gain versus $\sigma$ plane, with conditions as in Fig. 2. All modes have lower modal gain than the plane wave, as a result of the modulation depth and phase curvature induced by the boundaries. For a resonator with threshold gain of $30 \mathrm{~cm}^{-1}$, allowable modes must satisfy the steady-state condition $G_{m}=\Gamma g_{\mathrm{th}}\left(\Delta_{i}=0\right)$.

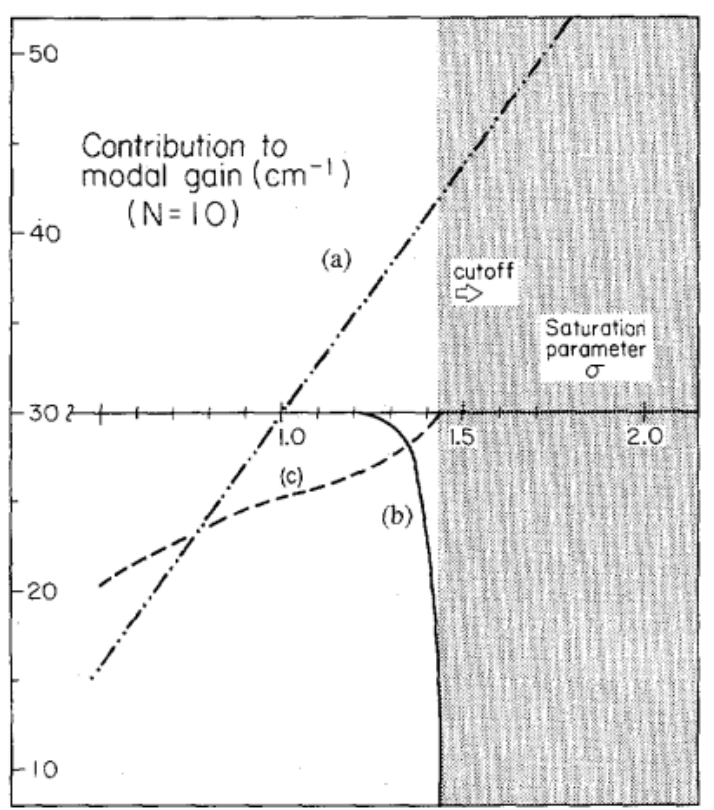

Fig. 4. Relative contribution to modal gain from (a) pumping $(\sigma-1)$, (b) modulation depth $(m)$, and (c) phase curvature $(\chi)$ for the $N=10$ filament solution in Fig. 3. Huge losses incurred by increasing modulation lead to an upper "cutoff" in $\sigma$. The $N=10$ solution does not exist beyond cutoff.

amount proportional to $m_{0}^{2}$. Secondly, as we have said, they impart an overall phase curvature to the field, which directs energy off of the axis of propagation and into the absorbing walls. The relative contributions of these two mechanisms are plotted in Fig. 4 for the lowest- $\sigma$ mode (i.e., the 10-filament mode). Their explicit contribution to the modal gain can be seen in (34)-(35). The two contributions combine to give each mode an upper cutoff in $\sigma$. As $\sigma$ increases (and thus power decreases), the modal gain for any given lateral mode will tend to increase as well, because the gain is becoming less and less saturated. However, the saturated filament spacing is becoming ever smaller, and the penalty that the mode pays is an increased modulation depth. Eventually, the modulation penalty overwhelms the benefits of gain saturation; the modal gain falls off again, and the cutoff appears. Fortunately, higher order modes (meaning more filaments) have successively higher cutoff- $\sigma$ 's, so higher modal gains are always possible with higher order modes.

We have made the assumption that, for a given $\Gamma g_{\text {th }}$, the field pattern with the greatest power (lowest $\sigma$ ) is the actual lasing mode. However, at a given threshold gain, solutions exist for all the lateral modes that are above cutoff. In Fig. 5 we plot the lateral power distribution for the 4 lowest order modes with modal gains of $30 \mathrm{~cm}^{-1}$. Successively higher order modes have more lobes, smaller modulation depth, and more phase curvature [as can be inferred from the correction to the filament wavenumber given in equation (25b)].

Finally, we comment briefly on the issue of modal selection. In linear theory, the rule is: the mode with the most gain wins. In a nonlinear system, the lasing mode is determined not by modal gain, but by a stability analysis. It is possible (but not likely) that the mode with the smallest $\sigma$ is unstable, while a mode with a higher $\sigma$ is stable. Alternatively, for certain operating points there may be multiple stable points (as is the case in coupled-cavity lasers, for example [31]).

The plausibility of the latter scenario may become more apparent by considering longitudinal variations within the laser. In a Fabry-Perot laser, the average optical intensity is not a constant, but is minimized somewhere between the two facets. Likewise, the material gain is maximized in the central region. According to our analysis, different modes may be favored in different regions of the resonator. The round-trip $\sigma$ of a lateral/longitudinal mode combination would thus be a weighted average of local $\sigma$, and this average would be the arbiter of which mode lases.

Concerning stability, we remark that in the unsaturated case where $|E(x)|^{2}<<E_{\text {sat }}^{2}$, the Helmholtz equation can be expressed in the form of the nonlinear Schrödinger equation. This equation is one of a class which admits soliton-like solutions, which are known to be unstable against many types of perturbation [32]. Thus, in the lowpower regime, the laser may exhibit instability. Above saturation intensity, however, the nonlinearity is much weaker and the self-guiding mechanism is stabilized.

Until recently, stable, uniform near-field patterns have not been commonly observed, as an excellent crystal growth over the entire laser is required to realize spatially uniform gain and index profiles. We can see from Fig. 3 that the different longitudinal modes differ in modal gain by only a few $\mathrm{cm}^{-1}$. Furthermore, in numerical calculations we have found that variations in local gain of a fraction of a $\mathrm{cm}^{-1}$ will disrupt the smooth lateral structure. To some degree, the effects of longitudinal propagation can smooth out the effects of inhomogeneities. Nevertheless, the broad area laser will remain extremely sensitive to such effects. Possibly, the destabilizing effect of material defects can be overcome by structures such as the recently demonstrated "controlled filament" laser [33]. In this structure, the effective mirror reflectivity is mod- 


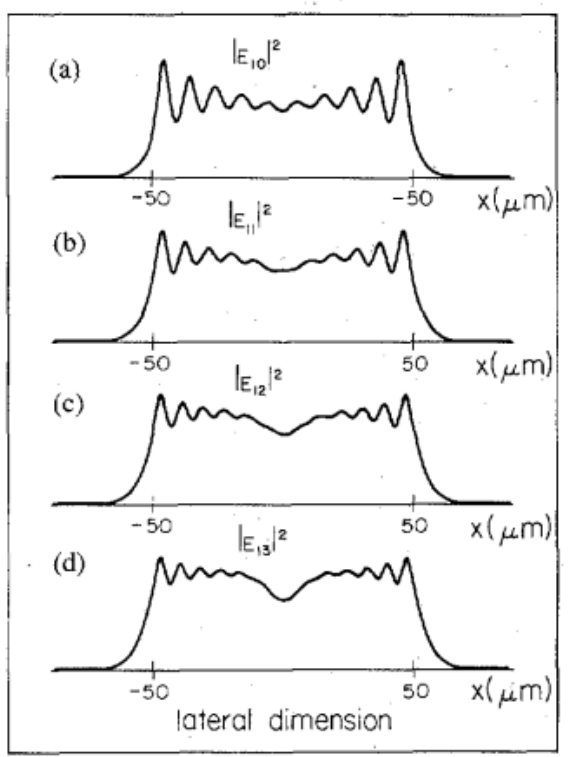

Fig. 5. Near-field intensities for the $N=10,11,12$, and 13 filament solutions for the $30 \mathrm{~cm}^{-1}$ loss resonator. Parts (a)-(d) correspond to modes A-D in Fig. 3.

ulated in the lateral dimension in order to induce filaments at prescribed positions. The present analysis indicates that this structure can be optimized if the reflectivity modulation is chosen with the same periodicity as the saturated filament spacing of the parent broad area laser.

\section{APPENDIX A}

This appendix details the solution of (18), (20), and (23). After dividing into real and imaginary parts, (18) becomes

$$
\begin{gathered}
a_{f}^{\prime \prime}+a_{s}^{\prime \prime}+\left(a_{f}^{\prime}+a_{s}^{\prime}\right)^{2}-\left(\theta_{f}+\theta_{s}\right)^{2} \\
+L_{0}^{r}+L_{1}^{r}\left(a_{f}+a_{s}\right)=0 \\
\theta_{f}^{\prime}+\theta_{s}^{\prime}+2\left(a_{f}^{\prime}+a_{s}^{\prime}\right)\left(\theta_{f}+\theta_{s}\right) \\
\quad+L_{0}^{i}+L_{1}^{i}\left(a_{f}+a_{s}\right)=0
\end{gathered}
$$

where, for example, $L_{0}^{r}$ denotes the real part of $L_{0}$. The fast variables $a_{f}, \theta_{f}$, are those which vary on a spatial scale of $O\left(\pi / q_{0}\right)$; the slow variables $a_{s}, \theta_{s}$ vary on a spatial scale of $O(1)$. To exploit the existence of two distinct length scales, we spatially average equations (A1) over a distance long compared to $2 \pi / q_{0}$, but short compared to 1 , leaving

$$
\begin{aligned}
a_{s}^{\prime \prime}+a_{s}^{\prime 2}-\theta_{s}^{2}+L_{0}^{r}+L_{1}^{r} a_{s}+\left\langle a_{f}^{\prime 2}-\theta_{f}^{2}\right\rangle=0 \\
\theta_{s}^{\prime}+2 a_{s}^{\prime} \theta_{s}+L_{0}^{i}+L_{1}^{i} a_{s}+2\left\langle a_{f}^{\prime} \theta_{f}\right\rangle=0 .
\end{aligned}
$$

$\langle\cdots\rangle$ denotes spatial averaging over the fast scale; (A2a)-(A2b) vary on the slow scale. They determine the slowly-varying quantities $a_{s}$ and $\theta_{s}$.

Keeping highest order terms (dropping $a_{s}^{\prime 2}, a_{s}^{\prime \prime}$ ) leads to the following two coupled equations for the slowlyvarying amplitude and phase gradient:

$$
\begin{array}{r}
-\theta_{s}^{2}+L_{0}^{r}+L_{1}^{r} a_{s}+\left\langle a_{f}^{\prime 2}-\theta_{f}^{2}\right\rangle=0 \\
\theta_{s}^{\prime}+2 a_{s}^{\prime} \theta_{s}+L_{0}^{i}+L_{1}^{i} a_{s}+2\left\langle a_{f}^{\prime} \theta_{f}\right\rangle=0 .
\end{array}
$$

Noting from (21b) that $L_{1}^{r}=b L_{1}^{i}$, we can eliminate $a_{s}$ easily from these equations, leaving the following equation in $\theta_{s}$ :

$$
b \theta_{s}^{\prime}+\theta_{s}\left(\theta_{s}+2 b a_{s}^{\prime}\right)=b^{2} \chi^{2}(\xi)
$$

where

$$
\begin{aligned}
b^{2} \chi^{2}(\xi) \equiv & L_{0}^{r}-b L_{0}^{i}+\left\langle a_{f}^{\prime 2}-\theta_{f}^{2}\right\rangle \\
& -2 b\left\langle a_{f}^{\prime} \theta_{f}\right\rangle .
\end{aligned}
$$

Neglecting $2 b a_{s}^{\prime}$ with respect to $\theta_{s}$, (A4) becomes

$$
b \theta_{s}^{\prime}=b^{2} \chi^{2}(\xi)-\theta_{s}^{2} \text {. }
$$

We choose initial conditions $a_{s}(0)=0, a_{s}^{\prime}(0)=0, \theta_{s}(0)$ $=0$, which together with (A3a) implies

$$
L_{0}^{r}+\left.\left\langle a_{f}^{\prime 2}-\theta_{f}^{2}\right\rangle\right|_{0}=0 \text {. }
$$

Then

$$
b \chi^{2}(0)=-L_{0}^{i}-\left.2\left\langle a_{f}^{\prime} \theta_{f}\right\rangle\right|_{0} .
$$

By approximating $\chi \simeq \chi(0) \equiv \chi_{0}$ (to be checked in Appendix B), we can integrate (A5) for the slowly-varying phase gradient, and then determine the slowly-varying amplitude from (A3a):

$$
\begin{aligned}
\theta_{s}= & b \chi_{0} \tanh \left(\chi_{0} \xi\right) \\
a_{s}= & \frac{1}{L_{1}^{r}}\left(\tanh ^{2}\left(\chi_{0} \xi\right)-L_{0}^{r}\right. \\
& \left.-\left\langle a_{f}^{\prime 2}-\theta_{f}^{2}\right\rangle\right) .
\end{aligned}
$$

We spatially-averaged (A1) to obtain the slow equations, (A2). Upon subtracting (A2) from (A1), we are left with the fast equations:

$$
\begin{aligned}
a_{f}^{\prime \prime}+ & \left(a_{f}^{\prime 2}-\left\langle a_{f}^{\prime 2}\right\rangle\right)+2 a_{f}^{\prime} a_{s}^{\prime} \\
& -\left(\theta_{f}^{2}-\left\langle\theta_{f}^{2}\right\rangle\right)-2 \theta_{f} \theta_{s}+L_{1}^{r} a_{f}=0 \\
\theta_{f}^{\prime}+ & 2\left(a_{f}^{\prime} \theta_{f}-\left\langle a_{f}^{\prime} \theta_{f}\right\rangle\right)+2 a_{f}^{\prime} \theta_{s} \\
& +2 a_{s}^{\prime} \theta_{f}+L_{1}^{i} a_{f}=0 .
\end{aligned}
$$

Equations (A9a)-(A9b) describe a harmonic oscillator system with a slowly-varying resonance frequency. The terms in parentheses act as driving terms at double the resoniant frequency; their corresponding response will be smaller than the fundamental, and we neglect them in favor of the fundamental response. Of the remaining terms, we keep only those of $O\left(q_{0}^{2}\right)$ and neglect the $O\left(q_{0}\right)$ and $O(1)$ terms. Equation (A9) then simplifies to

$$
\begin{aligned}
& a_{f}^{\prime \prime}-2 \theta_{s} \theta_{f}+L_{1}^{r} a_{f}=0 \\
& \theta_{f}^{\prime}+2 \theta_{s} a_{f}^{\prime}+L_{1}^{i} a_{f}=0 .
\end{aligned}
$$

In view of the slowly-varying coefficients, we form a WKB-type solution

$$
\begin{aligned}
& a_{f} \sim c_{1} e^{i\lceil q d \xi} \\
& \theta_{f} \sim c_{2} e^{i \int q d \xi}
\end{aligned}
$$


with slowly-varying $q(\xi)$. Together, (A10) becomes the following eigenvalue system

$$
\left[\begin{array}{cc}
-q^{2}+q_{0}^{2} & -2 \theta_{s} \\
\frac{q_{0}^{2}}{b}+i q 2 \theta_{s} & i q
\end{array}\right]\left(\begin{array}{l}
c_{1} \\
c_{2}
\end{array}\right)=\left(\begin{array}{l}
0 \\
0
\end{array}\right)
$$

The characteristic equation is

$$
q^{2}=q_{0}^{2}+4 \theta_{s}^{2}-i \frac{q_{0}^{2} 2 \theta_{s}}{b q} .
$$

Taking $q$ to be of the form $q_{r}-i q_{i}$, then $q^{2}=q_{r}^{2}-q_{i}^{2}-$ $i 2 q_{r} q_{i}$. Assuming $q_{r}^{2} \gg q_{i}^{2}$, we take

$$
q^{2}=q_{r}^{2}-i 2 q_{r} q_{i}
$$

Comparing (A13) and (A14) gives

$$
\begin{aligned}
& q_{r}^{2}=q_{0}^{2}+4 \theta_{s}^{2} \\
& q_{i}=\frac{\theta_{s}}{b} \frac{q_{0}^{2}}{q_{0}^{2}+4 \theta_{s}^{2}} .
\end{aligned}
$$

There are two solutions for $q_{r}$ (equal magnitude, opposite sign), and one for $q_{i}$. Thus the two solutions of (A13) for the propagation constant are

$$
q_{ \pm}= \pm q_{r}-i q_{i}
$$

where henceforth $q_{r}$ is understood to be the positive root of (A15a). Note that $q_{i}<<q_{r}$, as assumed. Now, $a_{f}$ and $\theta_{f}$ are real quantities. Symmetry dictates the choice of $a_{f}$ as

$$
\begin{aligned}
a_{f} & =\frac{m_{0}}{2}\left(e^{i\lceil q+d \xi}+e^{i \int q-d \xi}\right) \\
& =m_{0} e^{\int q_{i} d \xi} \cos \int q_{r} d \xi .
\end{aligned}
$$

The quantity $m(\xi) \equiv m_{0} \exp \int q_{i} d \xi$ is the slowly-varying modulation depth. The phase $\theta_{f}$ is then determined by the eigenvector of (A12). We have

$$
\left.\frac{c_{2}}{c_{1}}\right|_{ \pm}=i \frac{q_{0}^{2}}{b q_{ \pm}}-2 \theta_{s} \simeq \pm i \frac{q_{0}^{2}}{b q_{r}}-2 \theta_{s} .
$$

Then

$$
\begin{aligned}
\theta_{f} & =\frac{m_{0}}{2}\left[\left.\frac{c_{2}}{c_{1}}\right|_{+} e^{i j q+d \xi}+\left.\frac{c_{2}}{c_{1}}\right|_{-} e^{i j q-d \xi}\right] \\
& =m(\xi)\left(-\frac{q_{0}^{2}}{b q_{r}} \sin \int q_{r} d \xi-2 \theta_{s} \cos \int q_{r} d \xi\right) .
\end{aligned}
$$

Finally, let us estimate how the modulation depth and filament wavenumber vary across the half-width of the device. From (A15b) and (A8a) we have

$$
\begin{aligned}
\int q_{i} d \xi & =\frac{q_{0}^{2}}{b} \int \frac{\theta_{s}}{q_{0}^{2}+4 \theta_{s}^{2}} d \xi \\
& =\frac{1}{2 \rho} \ln \left(1+\rho \sinh ^{2}\left(\chi_{0} \xi\right)\right)
\end{aligned}
$$

where

$$
\rho \equiv 1+\frac{4 b^{2} \chi_{0}^{2}}{q_{0}^{2}} .
$$

Hence the modulation depth is

$$
m(\xi)=m_{0} e^{\left\lceil q_{i} d \xi\right.}=m_{0}\left(1+\rho \sinh ^{2}\left(\chi_{0} \xi\right)\right)^{1 / 2 \rho} .
$$

Thus, the modulation depth is at a minimum, $m_{0}$, at the center of the device, and increases monotonically towards the boundary. For the filament wave vector, we will find that $2 \theta_{s} \leq q_{0}$, so $q_{r}=\sqrt{q_{0}^{2}+4 \theta_{s}^{2}} \approx q_{0}+2 \theta_{s}^{2} / q_{0}$. Then

$$
\int q_{r} d \xi=q_{0} \xi+\frac{2 b^{2} \chi_{0}}{q_{0}}\left(\chi_{0} \xi-\tanh \left(\chi_{0} \xi\right)\right) .
$$

At the middle of the device, the wave vector is simply $q_{0}$ and it, too, increases monotonically towards the boundary.

Finally, recalling the slow equations (A3), we can now evaluate the averaged quantities that depend on the fast solutions $a_{f}$ and $\theta_{f}$ :

$$
\begin{aligned}
\left\langle\theta_{f}^{2}\right\rangle & =\frac{m^{2}}{2}\left(\frac{q_{0}^{2}}{b^{2}} \frac{q_{0}^{2}}{q_{0}^{2}+4 \theta_{s}^{2}}+4 \theta_{s}^{2}\right) \\
\left\langle a_{f}^{\prime 2}\right\rangle & =\frac{m^{2}}{2}\left(\frac{\theta_{s}^{2}}{b^{2}}\left(\frac{q_{0}^{2}}{q_{0}^{2}+4 \theta_{s}^{2}}\right)^{2}+q_{0}^{2}+4 \theta_{s}^{2}\right) \\
\left\langle a_{f}^{\prime} \theta_{f}\right\rangle & =\frac{m^{2}}{2}\left(\frac{q_{0}^{2}}{b}-\frac{2 \theta_{s}^{2}}{b} \frac{q_{0}^{2}}{q_{0}^{2}+4 \theta_{s}^{2}}\right) .
\end{aligned}
$$

This enables explicit evaluation of the slowly-varying amplitude; together (A23) and (A8b) give

$$
a_{s}=\frac{1}{q_{0}^{2}}\left[b^{2} \chi_{0}^{2} \tanh ^{2} \chi_{0} \xi-L_{0}^{r}-\frac{m^{2}}{2} q_{0}^{2}\right]
$$

where terms of relative order $1 / b^{2}$ have been neglected. This equation can be simplified further. By imposing the condition $a_{s}(0)=0$, we infer from (A24) that $L_{0}^{r}$ $=-\frac{1}{2} m_{0}^{2} q_{0}^{2}$. Equation (A24) becomes

$$
a_{s}=\frac{b^{2} \chi_{0}^{2}}{q_{0}^{2}} \tanh ^{2} \chi_{0} \xi-\frac{1}{2}\left(m^{2}(\xi)-m_{0}^{2}\right) .
$$

The last calculation we need is the quantity $\chi$. From (A4b) we have

$$
\begin{aligned}
b^{2} \chi^{2}= & L_{0}^{r}-b L_{0}^{i}+\frac{m^{2}}{2} \frac{q_{0}^{2}}{q_{0}^{2}+4 \theta_{s}^{2}} \\
& \cdot\left[\frac{\theta_{s}^{2} q_{0}^{2}}{b^{2}\left(q_{0}^{2}+4 \theta_{s}^{2}\right)}-q_{0}^{2}\left(1+\frac{1}{b^{2}}\right)\right] .
\end{aligned}
$$

To a good approximation (lowest order in $1 / b^{2}$ ),

$$
b^{2} \chi^{2} \simeq L_{0}^{r}-b L_{0}^{i}-\frac{m^{2}}{2} \frac{q_{0}^{4}}{q_{0}^{2}+4 \theta_{s}^{2}} .
$$




\section{APPENDIX B}

In the solution of (A5)

$$
b \theta_{s}^{\prime}=b^{2} \chi^{2}(\xi)-\theta_{s}^{2}
$$

we made the approximation that $\chi(\xi)$ was a constant, $\chi_{0}$. The actual expression for $\chi(\xi)$ is

$$
\begin{aligned}
\chi^{2}(\xi)-\chi_{0}^{2}= & \frac{1}{2 b^{2}}\left[\left(m_{0}^{2}-m^{2}(\xi)\right) q_{0}^{2}\right. \\
& \left.+m^{2}(\xi) q_{0}^{2}\left(\frac{4 \theta_{s}^{2}}{q_{r}^{2}}\right)\right] .
\end{aligned}
$$

While $\chi(\xi)=\chi_{0}$ at $\xi=0$, the difference could conceivably become significant when $m(\xi) \gg m_{0}$. We can make an estimate of the error by taking

$$
\theta_{s}=\theta_{s 0}+\theta_{s 1}, \quad \theta_{s 1} \ll \theta_{s 0},
$$

where $\theta_{s 0}$ satisfies (A8). Substituting (B3) into (B2) gives

$$
b \theta_{s 1}^{\prime}+2 \theta_{s 0} \theta_{s 1}+\theta_{s 1}^{2}=b^{2}\left(\chi^{2}(\xi)-\chi_{0}^{2}\right) \text {. }
$$

We neglect $\theta_{s 1}^{2}$ (we are assuming the error is small) and substitute (B1) for $\chi^{2}(\xi)-\chi_{0}^{2}$, to get

$$
\theta_{s 1}^{\prime}+\frac{2}{b} \theta_{s 0} \theta_{s 1}=\frac{q_{0}^{2}}{2 b^{2}}\left(m_{0}^{2}-m^{2}(\xi) \frac{q_{0}^{2}}{q_{r}^{2}}\right) \text {. }
$$

(B5) has the solution

$$
\begin{aligned}
\theta_{s 1}= & \frac{m_{0}^{2} q_{0}^{2}}{2 b} \operatorname{sech}^{2} \chi_{0} \xi \int_{0}^{\xi} d \zeta \cosh ^{2} \chi_{0} \zeta \\
& \cdot\left[1-\frac{q_{0}^{2}\left(1+\rho \sinh ^{2} \chi_{0} \zeta\right)^{1 / \rho}}{q_{0}^{2}+4 b^{2} \chi_{0}^{2} \tanh ^{2} \chi_{0} \zeta}\right] .
\end{aligned}
$$

The integral in (B6) does not have a simple closed-form expression, but we are only interested in determining the approximate size of $\theta_{s 1}$ relative to $\theta_{s 0}$. We asymptotically expand (B6) for large and small $\chi_{0}$, yielding

$$
\begin{aligned}
\frac{\theta_{s 1}}{\max \left(\theta_{s 0}\right)}= & \frac{\theta_{s 1}}{b \chi_{0}} \sim \frac{m_{0}^{2} q_{0}^{2}}{4 b^{2} \chi_{0}^{2}} \text { as } \chi_{0} \rightarrow \infty \\
& \frac{\theta_{s 1}}{b \chi_{0}} \sim-\frac{m_{0}^{2} q_{0}^{2}}{6 b} \chi_{0} \xi^{3} \text { as } \chi_{0} \rightarrow 0
\end{aligned}
$$

While $\theta_{s 1}$ undergoes a sign change at some intermediate value of $\chi_{0}$, neither expression becomes $O(1)$ for reasonable values of $m_{0}, q_{0}$, and $\chi_{0}$. Therefore, the original approximation is valid.

\section{REFERENCES}

[1] J. C. Dyment, "Hermite-Gaussian mode patterns in GaAs junction lasers," Appl. Phys. Lett., vol. 10, pp. 84-86, 1967.

[2] E. Kapon, J. Katz, and A. Yariv, "Supermode analysis of phaselocked arrays of semiconductor lasers," Opt. Lett., vol. 10, no. 4, pp. 125-127, 1984.

[3] J. Katz, E. Kapon, C. Lindsey, J. S. Smith, S. Margalit, U. Shreter, and A. Yariv, "Phase-locked semiconductor laser array with separate contacts," Appl. Phys. Lett., vol. 43, pp. 1257-1260, 1984.

[4] E. Kapon, C. Lindsey, J. Katz, S. Margalit, and A. Yariv, "Chirped arrays of diode lasers for supermode control," Appl. Phys. Lett., vol. 45 , pp. 200-202, 1984.
[5] D. Ackley, "Phase-locked injection laser arrays with non-uniform stripe spacing,"'Electron Lett., vol. 20, pp. 695-697, 1984.

[6] L. Figueroa, C. Morrison, H. D. Law, and F. Goodwin, "Twinchannel laser with high cw power and low beam divergence," J. Appl. Phys., vol. 56, pp. 3357-3359, 1984.

[7] Y. Twu, A. Dienes, S. Wang, and J. R. Whinnery, "High power coupled-ridge waveguide semiconductor laser arrays," Appl. Phys. Lett., vol. 45, pp. 709-711, 1984.

[8] S. Mukai, C. Lindsey, J. Katz, E. Kapon, Z. Rav-noy, S. Margalit, and A. Yariv, "Fundamental mode oscillation of a buried ridge waveguide laser arrays," Appl. Phys. Lett., vol. 45, pp. 834-835, 1984.

[9] C. Lindsey, E. Kapon, J. Katz, S. Margalit, and A. Yariv, "Singlecontact tailored gain phased array of semiconductor lasers," Appl. Phys. Lett., vol. 45, pp. 722-724, 1984.

[10] C. Lindsey, P. Derry, and A. Yariv, "Tailored gain broad area semiconductor laser with single-lobed diffraction-limited farfield pattern," Electron Lett., vol. 21, pp. 671-673, 1985.

[11] D. Welch, D. Scifres, P. Cross, H. King, W. Streifer, R. D. Burnham, J. Yaeli, and T. Paoli, "High power cw operation of phased array diode lasers with diffraction-limited output beam," Appl. Phys. Lett., vol. 47, pp. 1134-1136, 1985.

[12] L. Goldberg, H. F. Taylor, J. F. Welles, and D. Scifres, "Injection locking of coupled-stripe diode laser arrays," Appl. Phys. Lett., vol. 46 , pp. 236-238, 1985.

[13] J. P. Hohimer, A. Owyoung, and G. R. Hadley, "Single-channel injection locking of a diode laser array with a cw dye laser," Appl. Phys. Lett., vol. 47; pp. 1244-1246, 1985.

[14] J. Salzman, T. Venkatesan, R. Lang, M. Mittelstein, and A. Yariv, "Unstable resonator cavity semiconductor lasers," Appl. Phys. Lett., vol. 46, pp. 218-220, 1985.

[15] A. Larsson, M. Mittlestein, Y. Arakawa, and A. Yariv, "High efficiency broad area single quantum well lasers with narrow single-lobed farfield pattern prepared by MBE," Electron Lett., vol. 22, no. 2, pp. 79-81, 1986.

[16] M. Mittlestein, J. Salzman, A. Larsson, and A. Yariv, unpublished.

[17] G. H. B. Thompson, "A theory for filamentation in semiconductor lasers," Optoelectronics, vol. 4, pp. 257-310, 1972.

[18] F. R. Nash, "Mode guidance parallel to the junction plane of double heterostructure GaAs lasers," J. Appl. Phys., vol. 44, pp. 4696-4707, 1973.

[19] P. A. Kirkby, A. R. Goodwin, G. H. B. Thompson, and P. R. Selway, "Observations of self-focusing in stripe geometry semiconductor lasers and the development of a comprehensive model of their operation," IEEE J. Quantum Electron, vol. QE-13, pp. 705-719, 1977.

[20] R. Lang, "Lateral transverse mode instability and its stabilization in stripe-geometry injection lasers," IEEE J. Quantum Electron, vol. QE-15, pp. 718-726, 1979.

[21] S. Wang, C. Y. Chen, A. Liao, and L. Figueroa, "Control of mode behavior in semiconductor lasers," IEEE J. Quantum Electron, vol. QE-17, pp. 453-468, 1981.

[22] L. Figueroa, T. Holcomb, K. Burghard, D. Bullock, C. Morrison, L. Zinkiewicz, and G. A. Evans, "Modeling of the optical characteristics for twin-channel laser structures,"' IEEE J. Quantum Electron, vol. QE-22, pp. 2141-2149, 1986.

[23] W. Streifer and E. Kapon, "Application of the equivalent index method to DH diode lasers," Appl. Opt., vol. 18, no. 22, pp. 37243725,1979

[24] W. Tsang, "Extremely low threshold AlGaAs graded-index waveguide separate confinement heterostructure lasers grown by moleculat beam epitaxy," Appl. Phys. Lett., vol. 40, pp. 217-219, 1982.

[25] C. Bender and S. Orszag, Advanced Mathematical Methods for Scientists and Engineers. New York: McGraw-Hill, 1978, p. 499.

[26] A. Yariv, Optical Electronics, 3rd ed. New York: Holt, Rinehart and Winston, 1985 , p. 142.

[27] G. Stegeman and C. Seaton, "Nonlinear integrated optics," J. Appl. Phys., vol. 58, pp. R57-R78, 1985.

[28] N. Dutta, N. Olsson, and W. Tsang, "Carrier-induced refractive index change in AlGaAs quantum well lasers," Appl. Phys. Lett., vol. 45, pp. 836-837, 1984 .

[29] A. Arakawa and A. Yariv, "Theory of gain, modulation response, and spectral linewidth in AIGaAs quantum well lasers," IEEE $J$. Quantum Electron, vol. QE-21, no. 10, pp. 1666-1674, 1985.

[30] J. Buus, "Principles of semiconductor laser modelling," IEE Proc., vol. 132-J, no. 1 , pp. $42-51,1985$.

[31] R. J. Lang and A. Yariv, "Intermodal stability of a coupled-cavity laser," IEEE J. Quantum Electron, vol. QE-22, pp. 631-636, 1986. 
[32] M. Ablowitz and A. Segur, Solitons and the Inverse Scattering Transform. Studies in Applied Mathematics, Siam, 1981, pp. 271-273.

[33] J. Salzman, A. Larsson and A. Yariv, "Phase locked controlled filament laser,"' Appl. Phys. Lett., vol. 49, pp. 611-613, 1986.

David Mehuys (S'85), for a photograph and biography, see p. 787 of the June 1987 issue of this JOURNAL.

Robert J. Lang (S'83-M'86), for a photograph and biography, see p. 399 of the April 1987 issue of this Journal.

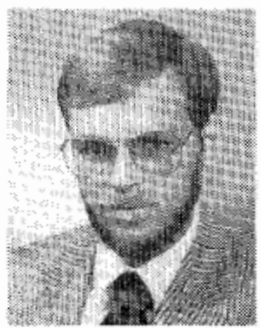

Michael Mittelstein was born in Hamburg, West Germany, in 1956. He received the Vordiploms (B.S. degrees) in physics and computer science, both in 1977, and the Diploma (M.S. degree) in physics in 1983, all from the University of Hamburg.

In 1981 he joined the Laser Physics Department under Prof. F.P. Schäfer, at the Max-PlanckInstitute of Biophysical Chemistry, Göttingen, West Germany. During the course of his work there he was exposed to several types of laser sys- tems and became specialized in the field of excimer lasers. Since 1983 he has been working towards the Ph.D. degree in applied physics with Prof. A. Yariv at the California Institute of Technology, Pasadena. He has been involved in various aspects of semiconductor lasers. After exploring unstable resonators and other configurations, he is currently investigating quantum well lasers. Major topics studied include nonlinear gain and the index of refraction, as well as spectral characteristics. He examines their effects on waveguiding and resonator requirements and their consequences on fundamental properties of high-performance devices.

Joseph Salzman, for a photograph and biography, see p. 400 of the April 1987 issue of this JOURNAL.

Amnon Yariv (S'56-M'59-F'70), for a photograph and biography, see p. 399 of the April 1987 issue of this JOURNAL. 